\title{
Potential New Approaches in Predicting Adverse Cardiac Events One Month after Major Vascular Surgery
}

\author{
Mladjan Golubovic ${ }^{a}$ Velimir Peric ${ }^{b}$ Dragana Stanojevic ${ }^{c}$ Milan Lazarevic ${ }^{d}$ \\ Nenad Jovanovic ${ }^{a}$ Nenad llic ${ }^{d}$ Miodrag Djordjevic ${ }^{e}$ Tomslav Kostic $^{\text {b, } c}$ \\ Dragan Milic b, d
}

${ }^{a}$ Clinic for Anesthesiology and Reanimatology, Clinical Center Nis, Nis, Serbia; ${ }^{b}$ Faculty of Medicine, University of Nis, Nis, Serbia; ${ }^{C}$ Clinic for Cardiology, Clinical Center Nis, Nis, Serbia; ${ }^{d}$ Clinic for Cardiovascular and Transplantation Surgery, Clinical Center Nis, Nis, Serbia; ${ }^{e}$ Clinic for General Surgery, Clinical Center Nis, Nis, Serbia

\section{Significance of the Study}

- The purpose of this study was to compare different cardiac risk scores in vascular elective surgery. The main finding of our study was that no risk factor tested individually had a statistically significant discriminatory ability. Combinations of traditional preoperative risk factors and scores can enhance the assessment of major adverse cardiac events in vascular surgery patients.

\section{Keywords}

Risk score · Risk assessment · Vascular surgery · Revised cardiac risk index $\cdot$ Cardiac biomarkers

\begin{abstract}
Objective: The aim of our study was to find the best model with sufficient power to improve the risk stratification in major vascular surgery patients during the first 30 days after this procedure. The discriminatory power of 4 biomarkers (troponin I [Tnl], N-terminal prohormone of brain natriuretic peptide [NT-proBNP], creatine kinase-MB isoenzyme [CK-MB], high-sensitivity C-reactive protein [hs-CRP]) was tested as well as 2 risk assessment models and 13 different combinations of them. Subjects and Methods: The study included 122 patients (77\% men, $23 \%$ women) with an average age of $67.03 \pm 4.5$ years. An aortobifemoral bypass was performed
\end{abstract}

\begin{tabular}{ll}
\hline KARGER & $\begin{array}{l}\text { (c) } 2018 \text { The Author(s) } \\
\text { Published by S. Karger AG, Basel }\end{array}$ \\
E-Mail karger@karger.com & $\begin{array}{l}\text { This is an Open Access article licensed under the Creative Commons } \\
\text { Attribution-NonCommercial-4.0 International License (CC BY-NC) } \\
\text { (http://www.karger.com/Services/OpenAccessLicense), applicable to } \\
\text { the online version of the article only. Usage and distribution for } \\
\text { commercial purposes requires written permission. }\end{array}$
\end{tabular}

in $6.56 \%$ of the patients, a femoropopliteal bypass in $18.85 \%$, and $49.18 \%$ received open surgical reconstruction of the carotid arteries. A total of $25.41 \%$ of the patients were given an aortobi-iliac bypass. Results: During the first 30 days, 13 patients (10.7\%) had 17 cardiac complications. The most common complication was the new onset of atrial fibrillation (35.3\%). During the first 10 days, 10 patients had 1 complication and 2 patients had 2 cardiac events, while 1 patient had 3 complications. By comparing combinations of scores and markers, it was shown that revised cardiac risk index (RCRI) + Vascular Portsmouth Physiological and Operative Severity Score (V-POSSUM) + hsTnl and RCRI + V-POSSUM + hsTnl + NT-proBNP with $100 \%$ sensitivity, $>80 \%$ specificity had the best discriminatory ability (AUC 0.924 and 0.933 , respectively; $p<0.001$ for both models) for cardiac complications during the 30 days after surgery. Conclusion: Combinations of traditional preoperative risk factors and scores can enhance 
the assessment of major adverse cardiac events (MACE) in patients preparing for large vascular surgery. Using only one risk score in these patients seems to be underperforming in preoperative risk assessment. @2018 The Author(s)

Published by S. Karger AG, Basel tested the discriminatory power of the four mentioned biomarkers (TnI, NT-proBNP, CK-MB, hs-CRP) included in the various pathophysiologic processes, two risk assessment models, as well as 13 different combinations of them.

\section{Subjects and Methods}

The study was approved by the Ethics Committee of the Faculty of Medicine, University of Nis, Nis, Serbia. During 2017, we enrolled 122 patients prepared for major open elective vascular surgery (abdominal aortic aneurysm repair, infrainguinal arterial reconstruction, or carotid endarterectomy) from the Clinic for Cardiothoracic Surgery and Transplantation, Clinical Center Nis, in the prospective trial. The exclusion criteria were: (1) age $<21$ years, (2) unstable coronary artery disease, and (3) decompensated heart failure. All surgical procedures were performed under general anesthesia. All patients initially underwent detailed evaluation of their medical history, a physical examination, routine hematologic and biochemical blood analysis, a 12-lead electrocardiogram, and chest radiography. Blood samples were taken within $48 \mathrm{~h}$ prior to surgery from the antecubital vein and stored in serum vacutainer tubes without additives. NT-proBNP $(\mathrm{pg} / \mathrm{mL})$ and $\mathrm{hsTnI}$ $(\mathrm{ng} / \mathrm{mL})$ levels were measured from the whole blood specimens using chemiluminescence enzyme immunoassay technology (CLEIA) and Magtration ${ }^{\circledR}$ technology on a PATHFAST Immunoanalyzer (Mitsubishi Chemical Europe GmbH, Düsseldorf, Germany). After centrifugation, the serum was separated and frozen at $-80^{\circ} \mathrm{C}$ until analysis. The hs-CRP $(\mathrm{mg} / \mathrm{L})$ and CK-MB (U/L) were measured in serum using the immunoturbidimetry method on a Beckman Coulter AU 680 analyzer (Beckman Coulter Inc., Brea, CA, USA). We used online risk calculator software to calculate the RCRI (www.mdcalc.com/revised-cardiac-risk-index-preoperative-risk) and V-POSSUM (http://www.riskprediction.org. uk/vasc-index.php). During the 30 days after the surgical procedure, we recorded MACE such as: acute myocardial infarction, ventricular arrhythmias, decompensated heart failure (NYHA 4; New York Heart Association Classification) [14], and a new onset of atrial fibrillation. All patients received optimal medical pre- and postoperative therapy for each cardiac disease or event prescribed by attending anesthesiologist and/or cardiologist according to ESC guidelines [2]. The most prescribed drugs were beta-blockers (73.8\%), ACE (angiotensin-converting enzyme) inhibitors (70.5\%), and antiplatelet drugs (64.8\%).

\section{Statistical Analysis}

The data obtained were entered into the database, arranged into tables, and shown graphically. As a type of descriptive statistics, the data were presented in the form of their arithmetic mean and standard deviation, median and interquartile differences, minimum and maximum values, and in the form of absolute or relative numbers. The normality of the data was tested using the Kolmogorov-Smirnov test. When comparing two data groups, if normal distribution was satisfied, a $t$ test was used; otherwise Mann-Whitney's U test was performed. When comparing three or more data sets, if normal distribution was satisfied, ANOVA was used, and the Tukey test was used for the post hoc analysis. If normal distribution was not satisfied when comparing three or more 
data sets, the Kruskal-Wallis test was used, in which case MannWhitney's U test was used as a post hoc analysis. To compare the attributes, a $\chi^{2}$ test or Fisher's exact probability test was used. Cox regression analysis was used to determine the hazard ratio (HR) for each of the risk factors investigated. Then, based on the NTproBNP and TnI values, the respondents were divided into two groups: those with a value in the upper quartile and others. ROC analysis was used to evaluate the model's discrimination. To construct a ROC curve for multiple variables from logistic regression analysis, a single variable is formed based on the probability of several individual variables. A comparison of multiple ROC curves was carried out using the DeLong test. Statistical data processing including descriptive statistics methods, Kaplan-Meier curves, Cox regression analysis, and ROC analysis was carried out in the SPSS 16.0 program package (SPSS Inc., Chicago, IL, USA). MedCalc version 18 was used to compare the ROC curves. Statistical significance was determined for a $p$ value $<0.05$.

\section{Results}

In this study, we included 122 patients (94 men [77.0\%], 28 women [23.0\%]) with an average age of $67.03 \pm 4.5$ years (min 48 , max 84 years). Regarding the cardiovascular risk factors, $85.2 \%$ (104) of our patients had arterial hypertension, $25.4 \%$ (31) had dyslipidemia, 31.1\% (38) insulin-independent diabetes mellitus, and 15.6\% (19) had insulin-dependent diabetes mellitus. Among the enrolled patients: $40.2 \%$ (49) were smokers and $40.2 \%$ (49) had a positive family history for cardiac diseases. Regarding the comorbidities, $21.3 \%$ (26) of the patients had coronary artery disease, $17.2 \%$ (21) had previous myocardial infarction, $1.6 \%$ (2) had prior surgical myocardial revascularization, $5.7 \%$ (7) had previous percutaneous coronary intervention, 9.8\% (12) had cardiomyopathy, 4.9\% (6) patients have atrial fibrillation, and $26.2 \%$ (32) had previous cerebrovascular insult.

Eight patients had open reconstruction of the aorta or aortobifemoral bypass $(6.56 \%)$, in 23 patients open reconstruction of the infrainguinal arteries was performed or femoropopliteal bypass (18.85\%), and in 60 patients open surgical reconstruction of the carotid arteries was carried out (49.18\%). In 31 patients, infrarenal aortic repair was performed (aortobi-iliac bypass [25.41\%]). The results of basic biochemical tests and NT-proBNP, cTnI, and hs-CRP measurements are presented in Table 1.

During the first 30 days, 13 patients $(10.7 \%)$ had 17 cardiac complications. The most common complication was the new onset of atrial fibrillation (35.3\%). Ten patients had 1 complication (76.9\%) and 2 patients had 2 cardiac events (15.4\%), while 1 patient had 3 complications $(7.7 \%)$. One patient died as a consequence of myo-

Predicting Adverse Cardiac Events after Major Vascular Surgery
Table 1. Basic biochemical parameters

\begin{tabular}{lccr}
\hline Parameter & Median \pm SD & \multicolumn{1}{l}{ Min } & \multicolumn{1}{c}{ Max } \\
\hline Hemoglobin & $16.35 \pm 17.33$ & 10.00 & 130.00 \\
Creatinine & $96.76 \pm 27.76$ & 56.40 & 236.00 \\
Leukocytes & $8.09 \pm 2.42$ & 3.00 & 17.00 \\
Platelets & $233.11 \pm 78.20$ & 17.00 & 552.00 \\
Urea & $6.53 \pm 2.18$ & 2.00 & 16.00 \\
hsCRP & $6.58 \pm 12.67$ & 0.00 & 105.00 \\
LDL cholesterol & $3.01 \pm 1.00$ & 1.00 & 5.80 \\
LVEF & $54.99 \pm 7.25$ & 38.00 & 76.00 \\
HDL cholesterol & $1.10 \pm 0.26$ & 0.52 & 2.00 \\
CK-MB & $107.93 \pm 115.51$ & 1.08 & 982.00 \\
hs-CRP & $3.28 \pm 15.16$ & 0 & 129.00 \\
NT-proBNP & $400.25 \pm 860.82$ & 2.00 & $6,682.00$ \\
CK-MB & $27.14 \pm 47.64$ & 0 & 488.00 \\
hsTnI & $0.002 \pm 0.005$ & 0.000 & 0.030 \\
P-SEP & $327.61 \pm 819.62$ & 102.00 & 6,502 \\
\hline
\end{tabular}

Min, minimal value; Max, maximal value; hsCRP, highsensitivity C-reactive protein; LDL, low-density cholesterol; LVEF, left ventricle ejection fraction; HDL, high-density cholesterol; CK$\mathrm{MB}$, creatine kinase-MB isoenzyme; NT-proBNP, N-terminal prohormone of brain natriuretic peptide; P-SEP, presepsin.

cardial infarction, 4 (23.5\%) had ventricular arrhythmia, $1(5.9 \%)$ had cardiopulmonary resuscitation, 5 (29.4\%) had decompensated heart failure, $6(35.3 \%)$ had new onset of atrial fibrillation. No pulmonary thromboembolism was detected in any of the patients.

Cardiac events during the first month after elective major surgery were not related to age, gender, atrial fibrillation, prior stroke, coronary artery disease, cardiomyopathy, prior percutaneous coronary intervention, previous myocardial infarction or surgical revascularization, hypertension, diabetes mellitus, dyslipidemia, smoking, or a family history of cardiac diseases. The average duration spent in an intensive care unit was significantly longer in patients with postoperative cardiovascular complications.

There was no relation between the type of surgery and postoperative complications. In both groups (with and without complications), a carotid endarterectomy was most often performed (38.5 and 50.5\%). Analysis of the ROC curve for individual markers showed no statistically significant discriminatory ability. By comparing combinations of scores and markers (Table 2; Fig. 1), it was seen that an RCRI with hsTnI had good discriminatory power (AUC 0.885, $p<0.001$ ). By adding the V-POSSUM to RCRI + hsTnI, we achieved a model with even better discriminatory ability compared to the previous combina- 


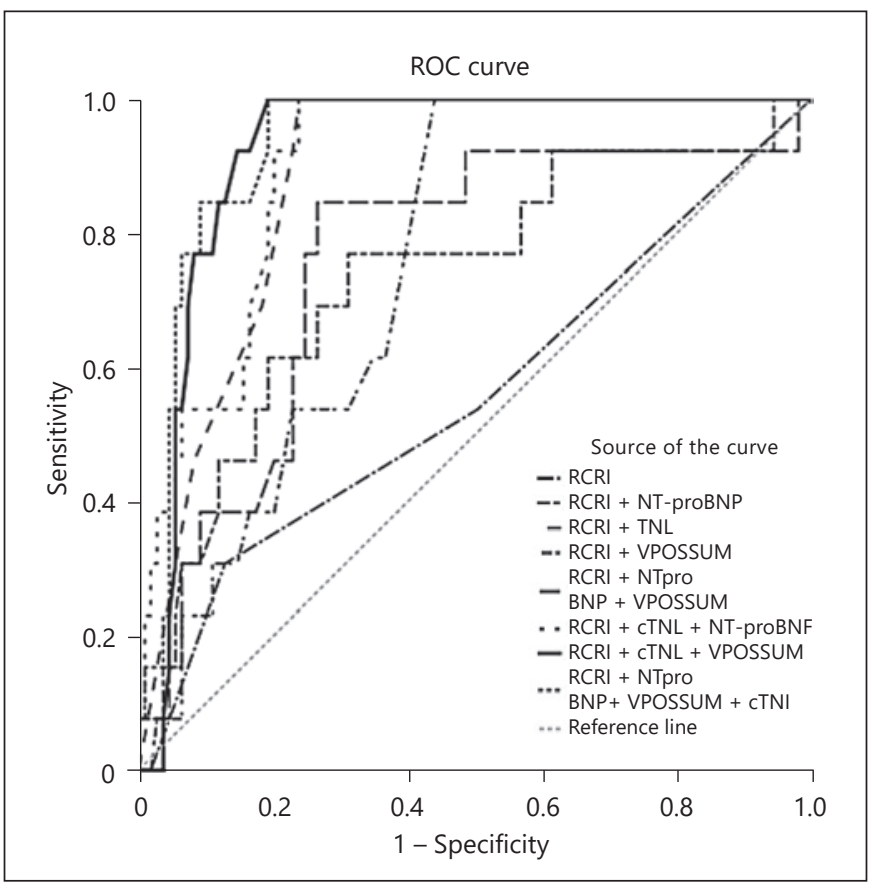

Fig. 1. ROC curves for various combinations of biomarkers in relation to the occurrence of cardiac complications during the first month.

tion of markers (AUC 0.924, $p<0.001$ ). By adding the NT-proBNP to RCRI + hsTnI + V-POSSSUM, the model with the best discriminatory ability was obtained (AUC $0.933, p<0.001)$.

Comparison of the ROC curves for different combinations showed that there is a statistically significant difference between the following combinations: RCRI + hsTnI versus RCRI + V-POSSUM $(\triangle \mathrm{AUC}=0.131, p=0.007)$, RCRI + V-POSSUM versus RCRI + NT-proBNP + V-POSSUM + hsTnI $(\triangle \mathrm{AUC}=0.179, p<0.001), \mathrm{RCRI}+\mathrm{V}$-POSSUM versus RCRI + hsTnI + NT-proBNP $(\triangle A U C=0.145$, $p=0.005), \mathrm{RCRI}+\mathrm{V}$-POSSUM versus RCRI $+\mathrm{hsTnI}+\mathrm{V}$ POSSUM $(\triangle \mathrm{AUC}=0.170, p<0.001), \mathrm{RCRI}+\mathrm{NT}-$ pro$\mathrm{BNP}+\mathrm{V}$-POSSUM versus RCRI + NT-proBNP + V-POS$\mathrm{SUM}+\mathrm{hsTnI}(\triangle \mathrm{AUC}=0.175, p=0.008), \mathrm{RCRI}+\mathrm{NT}-$ proBNP + V-POSSUM versus RCRI + hsTnI + V-POSSUM $(\triangle \mathrm{AUC}=0.165, p=0.026)$. There were no statistically significant differences between the other combinations.

All combinations of biomarkers had statistically significant discriminatory power with AUC $>0.8$ (Table 3; Fig. 2). Excellent discriminatory ability for determining cardiac complications in the first month was achieved by hsTnI + NT-proBNP + V-POSSUM (AUC 0.916, $p<$ 0.001). Comparison of the ROC curves showed that add-

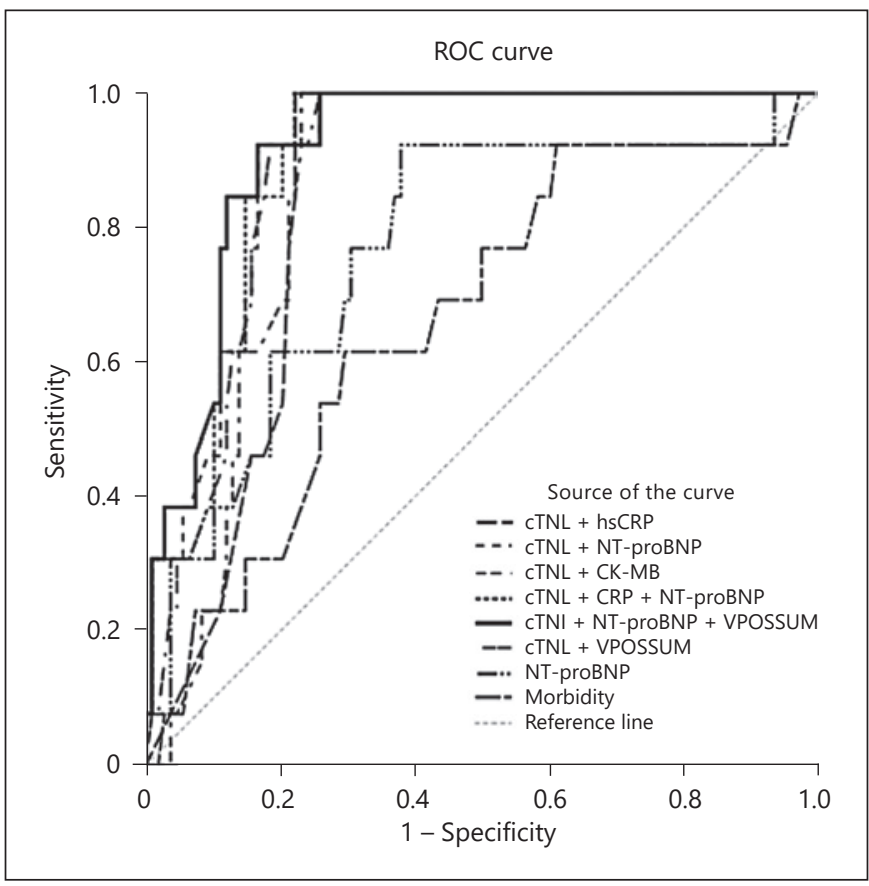

Fig. 2. ROC curves for various combinations of biomarkers in comparison to the occurrence of cardiac complications during the first month.

ing hsTnI to NT-proBNP significantly improved the discriminatory ability of the model ( $\triangle$ AUC $0.132, p=0.016)$. The high-sensitivity TnI + hs-CRP + NT-proBNP model had a statistically significantly better discriminatory ability compared to NT-proBNP ( $\triangle$ AUC $0.132, p=0.020)$. The high-sensitivity TnI + NT-proBNP + V-POSSUM model had a statistically significantly better discriminatory ability than the hsTnI + hs-CRP model ( $\triangle \mathrm{AUC} 0.073$, $p=0.045$ ).

The following models had a statistically significant better discriminatory ability in comparison to the individual discriminatory ability of V-POSSUM: hsTnI + hsCRP $(\triangle \mathrm{AUC} 0.182, p=0.028)$, hsTnI + NT-proBNP $(\triangle \mathrm{AUC} 0.240, p=0.002)$, hsTnI $+\mathrm{CRP}+\mathrm{NT}$-proBNP $(\triangle \mathrm{AUC} 0.239, p=0.003)$, hsTnI $+\mathrm{NT}$-proBNP + V-POSSUM ( $\triangle$ AUC 0.254, $p<0.001)$, hsTnI + V-POSSUM $(\triangle \mathrm{AUC} 0.228, p<0.001)$.

\section{Discussion}

The purpose of this study was to compare different cardiac risk scores in vascular elective surgery in a large single-institute patient population. The incorporation of 
Table 2. Discriminatory ability of different combinations of biomarkers and clinical scores

\begin{tabular}{lrrrrrr}
\hline Combinations of markers and scores & SEN & SPEC & AUC & SE & 95\% CI & $p$ \\
\hline RCRI & 30.8 & 87.2 & 0.557 & 0.092 & $0.376-0.738$ & 0.502 \\
RCRI + NT-proBNP & 76.9 & 68.8 & 0.735 & 0.079 & $0.581-0.889$ & $0.006^{*}$ \\
RCRI + hsTnI & 100.0 & 76.1 & 0.885 & 0.032 & $0.822-0.947$ & $0.000^{*}$ \\
RCRI + V-POSSUM & 53.8 & 77.1 & 0.754 & 0.051 & $0.654-0.855$ & $0.003^{*}$ \\
RCRI + NT-proBNP + V-POSSUM & 84.6 & 72.5 & 0.758 & 0.073 & $0.615-0.902$ & $0.002^{*}$ \\
RCRI + hsTnI + NT-proBNP & 92.3 & 79.8 & 0.899 & 0.031 & $0.837-0.960$ & $0.000^{*}$ \\
RCRI + hsTnI + V-POSSUM & 100.0 & 80.7 & 0.924 & 0.024 & $0.876-0.972$ & $0.000^{*}$ \\
RCRI + NT-proBNP + V-POSSUM + hsTnI & 100.0 & 80.7 & 0.933 & 0.024 & $0.886-0.980$ & $0.000^{*}$ \\
\hline
\end{tabular}

* Statistically significant at $p<0.05$.

Table 3. ROC curve for postoperative cardiac events

\begin{tabular}{lrrrrrc}
\hline Combinations of scores and markers & SEN & SPEC & AUC & SE & $95 \%$ CI & $p$ \\
\hline NT-proBNP & 76.9 & 69.4 & 0.769 & 0.071 & $0.630-0.907$ & $<0.001^{*}$ \\
V-POSSUM & 61.5 & 70.4 & 0.663 & 0.077 & $0.511-0.814$ & 0.056 \\
hsTnI + hsCRP & 100.0 & 77.8 & 0.843 & 0.035 & $0.773-0.912$ & $<0.001^{*}$ \\
hsTnI + NT-proBNP & 92.3 & 83.3 & 0.901 & 0.030 & $0.842-0.960$ & $<0.001^{*}$ \\
hsTnI + CK-MB & 100.0 & 76.9 & 0.854 & 0.034 & $0.788-0.920$ & $<0.001^{*}$ \\
hsTnI + V-POSSUM & 92.3 & 81.5 & 0.889 & 0.031 & $0.829-0.949$ & $<0.001^{*}$ \\
hsTnI + CRP + NT-proBNP & 84.6 & 85.2 & 0.901 & 0.030 & $0.842-0.959$ & $<0.001^{*}$ \\
hsTnI + NT-proBNP + V-POSSUM & 92.3 & 83.3 & 0.916 & 0.028 & $0.861-0.970$ & $<0.001^{*}$ \\
\hline
\end{tabular}

* Statistically significant at $p<0.05$.

biomarkers which reflect myocardial injury, inflammation, and myocardial wall stress into well-known risk models significantly improved stratification for MACE.

The results show that five algorithms had high accuracy in the short-term estimation of cardiac complications. During postoperative monitoring, all cardiac complications were recorded. The absence of consensus for defining MACE is a major obstacle in comparing the results with other studies that explore the same problem [15].

The ROC curve (c-statistic) is a good statistical tool for determining the performance of risk factors as MACE predictors [16]. AUC $>90 \%$ and $p<0.001$ were regarded as having significant discriminatory power with statistical significance.

The main finding of our study is that no risk factor tested individually had a statistically significant discriminatory ability. We suggest that the lack of efficiency of the V-POSSUM score could be attributed to the previously observed geographic variations in the potency of this score [6]. Many previous studies have suggested that RCRI has lower discriminatory potential for estimating adverse cardiac events and that it may be more useful in excluding low-risk patients [17]. RCRI is limited in terms of the identification of patients at high cardiac risk in real clinical settings $[18,19]$. In a study of 467 patients, Bae et al. [20] showed that about $80 \%$ of patients with perioperative MACE were classified as being in the low- or intermediate-risk groups.

Assays for hsTnI from blood samples can detect values $<0.020 \mathrm{ng} / \mathrm{mL}$, which is the upper referent limit (99th percentile). The lowest concentration of TnI with a coefficient of variation $(\mathrm{CV}) \leq 10 \%$ within the limit of quantification (LoQ) was $0.0031 \mathrm{ng} / \mathrm{mL}$ [21]. Given that the LoQ of troponin assays may be equal or higher but cannot be lower than the limit of detection (LoD) [22], and the reported value of LoD for TnT was $5 \mathrm{ng} / \mathrm{L}$ [23], we chose to investigate the influence of preoperative TnI concentration and not TnT which was used more often for this purpose. 
Two models showed particularly good characteristics: RCRI + V-POSSUM + hsTnI and RCRI + V-POSSUM + hsTnI + NT-proBNP with 100\% sensitivity, >80\% specificity and AUC 92.4 and $93.3 \%$, respectively. Other studies have confirmed the potential predictive value of combinations of risk scores in surgical patients. Park et al. [24] highlighted the significance in the prediction of shortterm MACE for a combination of NT-proBNP, RCRI, and echocardiographic parameters in major noncardiac surgery. A combination of hs-CRP and NT-proBNP led to an increase of $5 \%$ in AUC and improved the prediction of postoperative cardiac events, although this differs from our study [25]. The addition of hsTnT and NT-proBNP improved the medium quality of RCRI in the estimation of postoperative myocardial infarction in noncardiac surgery by increasing the AUC by $12.6 \%$ [26]. A study that defined MACE in a similar way to our study demonstrated the low accuracy and underestimation of MACE by RCRI and another specific risk model for vascular surgery [27]. Although we were not able to demonstrate the importance of individual markers in the prediction of postoperative cardiovascular events, in a recently published study, we showed the importance of midregional proadrenomedulin as a single predictive marker measured preoperatively $[28,29]$. It is difficult to create a unique way of determining the risk score in patients preparing for major vascular surgery if a large number of events are followed in the postoperative course. In the future, we need to evaluate the models tested in our study for the prediction of short-term mortality, but a larger sample is needed.

\section{Conclusion}

Combinations of traditional preoperative risk factors and scores can enhance the assessment of major adverse cardiac events in patients who are preparing for a large vascular surgery. Those patients usually have coronary artery disease or at least traditional risk factors for it. The use of a single risk score in these patients seems to be underperforming in preoperative risk assessment. However, a combination could be a better way to predict the 30-day outcome in elective vascular surgery.

\section{Acknowledgement}

The authors wish to thank the research nurses Zorica Lazarevic and Stana Ilic without whose efforts this study would not have been possible.

\section{Disclosure Statement}

The authors have no conflicts of interest to declare.

\section{References}

1 Hertzer NR, Beven EG, Young JR, O’Hara PJ, Ruschhaupt WF 3rd, Graor RA, et al. Coronary artery disease in peripheral vascular patients. A classification of 1000 coronary angiograms and results of surgical management. Ann Surg. 1984 Feb;199(2):223-33.

2 Kristensen SD, Knuuti J, Saraste A, Anker S, Bøtker HE, De Hert S, et al.; Authors/Task Force Members. 2014 ESC/ESA Guidelines on non-cardiac surgery: cardiovascular assessment and management: The Joint Task Force on non-cardiac surgery: cardiovascular assessment and management of the European Society of Cardiology (ESC) and the European Society of Anaesthesiology (ESA). Eur J Anaesthesiol. 2014 Oct;31(10):517-73.

3 Fleisher LA, Fleischmann KE, Auerbach AD, Barnason SA, Beckman JA, Bozkurt B, et al. 2014 ACC/AHA guideline on perioperative cardiovascular evaluation and management of patients undergoing noncardiac surgery: executive summary: a report of the American College of Cardiology/American Heart Asso- ciation Task Force on practice guidelines. J Nucl Cardiol. 2015 Feb;22(1):162-215.

4 Bryce GJ, Payne CJ, Gibson SC, Kingsmore DB, Byrne DS, Delles C. Risk stratification scores in elective open abdominal aortic aneurysm repair: are they suitable for preoperative decision making? Eur J Vasc Endovasc Surg. 2012 Jul;44(1):55-61.

5 Harris JR, Forbes TL, Steiner SH, Lawlor DK, Derose G, Harris KA. Risk-adjusted analysis of early mortality after ruptured abdominal aortic aneurysm repair. J Vasc Surg. 2005 Sep; 42(3):387-91

6 Tang TY, Walsh SR, Prytherch DR, Wijewardena C, Gaunt ME, Varty K, et al. POSSUM models in open abdominal aortic aneurysm surgery. Eur J Vasc Endovasc Surg. 2007 Nov; 34(5):499-504.

7 Kim LJ, Martinez EA, Faraday N, Dorman T, Fleisher LA, Perler BA, et al. Cardiac troponin I predicts short-term mortality in vascular surgery patients. Circulation. 2002 Oct; 106(18):2366-71.
8 Rodseth RN, Padayachee L, Biccard BM. A meta-analysis of the utility of pre-operative brain natriuretic peptide in predicting early and intermediate-term mortality and major adverse cardiac events in vascular surgical patients. Anaesthesia. 2008 Nov;63(11):122633.

9 Belluardo P, Cataliotti A, Bonaiuto L, Giuffrè E, Maugeri E, Noto P, et al. Lack of activation of molecular forms of the BNP system in human grade 1 hypertension and relationship to cardiac hypertrophy. Am J Physiol Heart Circ Physiol. 2006 Oct;291(4):H1529-35.

10 de Winter RJ, Koster RW, Sturk A, Sanders GT. Value of myoglobin, troponin T, and CKMBmass in ruling out an acute myocardial infarction in the emergency room. Circulation. 1995 Dec;92(12):3401-7.

11 Choi JH, Cho DK, Song YB, Hahn JY, Choi S, Gwon HC, et al. Preoperative NT-proBNP and CRP predict perioperative major cardiovascular events in non-cardiac surgery. Heart. 2010 Jan;96(1):56-62. 
12 Martins OM, Fonseca VF, Borges I, Martins V, Portal VL, Pellanda LC. C-Reactive protein predicts acute myocardial infarction during high-risk noncardiac and vascular surgery. Clinics (Sao Paulo). 2011;66(5):773-6.

13 Baron DM, Hochrieser H, Posch M, Metnitz B, Rhodes A, Moreno RP, et al.; European Surgical Outcomes Study (EuSOS) group for Trials Groups of European Society of Intensive Care Medicine; European Society of Anaesthesiology. Preoperative anaemia is associated with poor clinical outcome in non-cardiac surgery patients. Br J Anaesth. 2014 Sep; 113(3):416-23.

14 Biccard BM, Rodseth RN. Utility of clinical risk predictors for preoperative cardiovascular risk prediction. Br J Anaesth. 2011 Aug; 107(2):133-43.

15 The Criteria Committee of the New York Heart Association. Nomenclature and Criteria for Diagnosis of Diseases of the Heart and Great Vessels. 9th ed. Boston (Mass): Little, Brown \& Co; 1994. pp. 253-6.

16 Pencina MJ, D'Agostino RB Sr, D'Agostino RB Jr, Vasan RS. Evaluating the added predictive ability of a new marker: from area under the ROC curve to reclassification and beyond. Stat Med. 2008 Jan;27(2):157-72.

17 Ford MK, Beattie WS, Wijeysundera DN. Systematic review: prediction of perioperative cardiac complications and mortality by the revised cardiac risk index. Ann Intern Med. 2010 Jan;152(1):26-35.
18 Kim SE, Park DG, Lee JH, Han KR, Oh DJ. Utility of B-type natriuretic Peptide for predicting perioperative cardiovascular events in patients without history of cardiovascular disease undergoing major non-cardiac surgery. Korean Circ J. 2011 Jan;41(1):11-5.

19 Parmar CD, Torella F. Prediction of major adverse cardiac events in vascular surgery: are cardiac risk scores of any practical value? Vasc Endovascular Surg. 2010 Jan;44(1):14-9.

20 Bae MH, Jang SY, Choi WS, Kim KH, Park $\mathrm{SH}$, Lee JH, et al. A new revised cardiac risk index incorporating fragmented QRS complex as a prognostic marker in patients undergoing noncardiac vascular surgery. Am J Cardiol. 2013 Jul;112(1):122-7.

21 21st International Congress of Clinical Chemistry and Laboratory Medicine, IFCCWorldLab - EuroMedLab, Berlin, 15-19 May, 2011.

22 Armbruster DA, Pry T. Limit of blank, limit of detection and limit of quantitation. Clin Biochem Rev. 2008 Aug;29 Suppl 1:S49-52.

23 Body R, Burrows G, Carley S, Cullen L, Than $\mathrm{M}$, Jaffe AS, et al. High-sensitivity cardiac troponin $t$ concentrations below the limit of detection to exclude acute myocardial infarction: a prospective evaluation. Clin Chem. 2015 Jul;61(7):983-9.

24 Park SJ, Choi JH, Cho SJ, Chang SA, Choi JO, Lee SC, et al. Comparison of transthoracic echocardiography with $\mathrm{N}$-terminal pro-brain natriuretic Peptide as a tool for risk stratification of patients undergoing major noncardiac surgery. Korean Circ J. 2011 Sep;41(9):50511.
25 Goei D, Hoeks SE, Boersma E, Winkel TA, Dunkelgrun M, Flu WJ, et al. Incremental value of high-sensitivity C-reactive protein and $\mathrm{N}$-terminal pro-B-type natriuretic peptide for the prediction of postoperative cardiac events in noncardiac vascular surgery patients. Coron Artery Dis. 2009 May;20(3):219-24.

26 Kopec M, Duma A, Helwani MA, Brown J Brown F, Gage BF, et al. Improving prediction of postoperative myocardial infarction with high-sensitivity cardiac troponin T and NTproBNP. Anesth Analg. 2017 Feb;124(2): 398-405.

27 Gualandro DM, Puelacher C, LuratiBuse G, Llobet GB, Yu PC, Cardozo FA, et al.; GREAT network. Prediction of major cardiac events after vascular surgery. J Vasc Surg. 2017 Dec; 66(6):1826-1835.e1.

28 Golubović M, Janković R, Sokolović D, Ćosić V, Maravić-Stojkovic V, Kostić T, et al. Preoperative midrregional pro-adrenomedulin and high-sensitivity troponin $\mathrm{T}$ predict perioperative cardiovascular events in noncardiac surgery. Med Princ Pract. 2018;27(3):278-84.

29 Golubovic M, Stanojevic D, Jovanovic N, et al Mid-regional pro-adrenomedulin as a marker of perioperative mortality in non-cardiac surgery. Vojnosanitetski pregled. Military-medical and pharmaceutical review. 2018; DOI: https://doi.org/10.2298/VSP180522105G. 